\title{
Periodic Ripples in a free Smectic A Surface Skin
}

\author{
Fred Fischer \\ Physikalisches Institut, Westfälische Wilhelms-Universität, Münster \\ (Z. Naturforsch. 32 a, 429-431 [1977]; received March 19, 1977)
}

\begin{abstract}
A smectic A skin in a homeotropic texture on a liquid surface should form a periodic ripple when a horizontal compressive strain exceeds a critical value. The ripple wavelength is proportional to the fourth root of the skin thickness. In all practical cases the critical strain should be inversely proportional to the skin thickness. For a skin thickness approaching molecular dimensions, the critical strain has to be kept low by a small surface tension in order to avoid the formation of dislocations.
\end{abstract}

A solid elastic skin laid on the horizontal surface of a liquid aquires a periodic ripple formation when a horizontal compressive strain exceeds a critical value ${ }^{1}$. The ripple amplitude grows with the square root of the excess strain. This instability can be interpreted as a kind of second order phase transition. The ripple wavelength is proportional to the $3 / 4$ th power of the skin thickness. This result suggests that the same considerations apply to a smectic A skin which has its molecular layers parallel to the plane surface of the isotropic liquid.

From a smectic A layer with the same homeotropic texture, strongly anchored between parallel plane plates, one already knows some transitions induced by external fields. This so called HelfrichHurault effect ${ }^{2}$ describes the formation of an internal periodic deformation along the layer plane. It can be caused either by a critical magnetic field parallel to the layer $\left(\chi_{\mathrm{a}}>0\right)^{2}$ or by tension normal to the plates ${ }^{3}$. A related thermo-optical effect observed by Kahn ${ }^{4}$ produces the needed underpressure by suddenly cooling the layer, after it has been heated by light absorption. In all these cases, the ripple wavelength is proportional to the square root of the layer thickness.

The effect to be described in this paper differs from those named before in so far as no solid walls exist to which the smectic A skin is fixed. This will result in another power law between the ripple wavelength and the skin thickness. The results, however, are comparable to those obtained from a solid elastic skin.

In ortogonal coordinates (Fig. 1 a), the smectic A skin has the thickness $d$ in the $z$ direction and the length $L$ in the $x$ direction. It undergoes a compres-

Reprint requests to Prof. Dr. F. Fischer, Physikalisches Institut der Universität, Schloßplatz 7, D-4400 Münster. sive strain $\Delta L$ in the $x$ direction. The $y$ dimension may be kept constant. The displacement vector $\left(u_{x}, 0, u_{z}\right)$ has a zero $y$ component and the other components do not depend on $y$. The strain may be applied in an adiabatic way so that the number of molecular layers remains unchanged. They only increase their thickness and may undergo an $x$ dependent modulation. This can be described by the $z$ and $x$ dependence of $u_{z}$. In an approximation where the density of the smectic A liquid crystal remains constant, one obtains the following expression for the free energy density $F^{5}$ :

$$
F=\frac{1}{2} \bar{B} u_{z, z}^{2}+\frac{1}{2} K_{1} u_{z, x x}^{2} .
$$

$\bar{B}$ is an elastic module which characterizes the resistance of a molecular skin against a change in thickness. $K_{1}$ is the elastic splay constant. In this approximation we are not able to describe the strain induced rippling. As already shown in other examples ${ }^{6}$ one has to replace $u_{z, z}=\Delta a_{0} / a_{0}$ (see Fig. $1 \mathrm{~b})$ by $\Delta a / a_{0}=\Delta a_{0} / a_{0}-\frac{1}{2} u_{z, x^{2}}$. This ap-

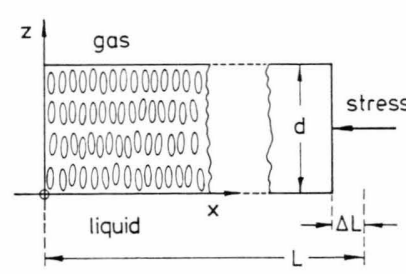

(a)

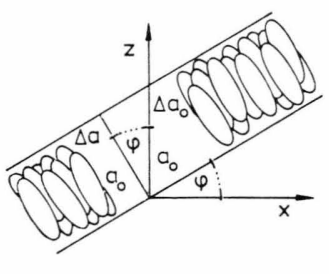

(b)
Fig. 1. a) Coordinate system for the smectic A skin on the liquid surface. $\Delta L / L$ is the applied compressive strain. b) Molecular layer in oblique orientation. $\Delta a$ is the actual increase of the original thickness $a_{0}$.

proximation still holds for small angels $\varphi \cdot a_{0}$ is the thickness of an undeformed molecular layer. Because of the incompressibility assumed for the smectic A 
skin $\Delta a_{0} / a_{0}$ comes out to be equal to $(\Delta L / L)$. From the modified Eq. (1) and by adding the terms for gravity and for surface tensions we obtain the free energy per surface area

$$
\begin{aligned}
f(x) & =\frac{1}{2} \bar{B} d\left(\Delta L / L-\frac{1}{2} u_{z, x}^{2}\right)^{2}+\frac{1}{2} K_{1} d u_{z, x x}^{2} \\
& +\frac{1}{2} \varrho g u_{z}^{2}+\frac{1}{2} \gamma u_{z, x}^{2} .
\end{aligned}
$$

$\varrho$ means the density of the isotropic liquid (or when stratified with another liquid, the difference in densities) and $\gamma$ means the sum of surface tensions of both the upper and lower side of the skin. On account of the absence of solid walls there is no $z$ dependence of $f$.

Actually one should apply variational methods to minimize $f(x)$. This would give a nonlinear differential equation of fourth order in $u_{z}$. However, near the expected 'phase transition', that means for small ripple amplitudes, we may use the harmonic approximation

$$
u_{z}=\zeta_{0} \cos k x
$$

in Equation (2). We may average $f(x)$ over one wavelength $\lambda=2 \pi / k$ and by using $\left\langle\sin ^{2} k x\right\rangle=$ $\left\langle\cos ^{2} k x\right\rangle=1 / 2$ and $\left\langle\sin ^{4} k x\right\rangle=3 / 8$ we obtain the mean free energy per unit-area in Landau's meaning

$$
f=f_{0}+f_{\mathrm{II}} \zeta_{0}{ }^{2}+f_{\mathrm{IV}} \zeta_{0}^{4}
$$

where the coefficients have the following form:

$$
\begin{aligned}
f_{0} & =\frac{1}{2} \bar{B} d(\Delta L / L)^{2}, \\
f_{\mathrm{II}} & =\frac{1}{4}\left[-\bar{B} d(\Delta L / L) k^{2}+K_{1} d k^{4}+\varrho g+\gamma k^{2}\right], \\
f_{\mathrm{IV}} & =\frac{3}{64} \bar{B} d k^{4} .
\end{aligned}
$$

A comparison of Eqs. (5) and (6) with the corresponding expressions for the solid skin ${ }^{1}$ shows a completely analogous form. One only has to replace $\bar{B}$ by the Young modulus $E$ of the solid skin and $K_{1}$ by $E d^{2} / 12$. In our approximation a single molecular smectic A $\operatorname{skin}\left(d=a_{0}\right)$ behaves like a solid elastic skin of the same thickness. And a smectic A skin of thickness $d=n a_{0}$ behaves like a file of $n$ solid layers of thickness $a_{0}$ freely sliding face by face. A vivid example of the reduced bending stiffness is given by a file of papers, compared with a wooden plate of the same size. Hence in the approximation used here we should get

$$
K_{1} / \bar{B}=a_{0}{ }^{2} / 12 .
$$

However one knows that when approaching the smectic A-nematic transition point $\bar{B}$ will go to zero. So Eq. (8) might be only useful sufficiently away from this transition point.

The further steps are the same as for the solid skin. For a better understanding, the following analogy to the ferromagnetic phase transition may help: $(\Delta L / L)$ behaves in the same way as a reduction in temperature $-\Delta T ; \zeta_{0}$ the same as the spontaneous magnetisation $M$, and hence it can be used as an order parameter. Because $f_{I V}>0$ we need not consider higher powers in $\zeta_{0}{ }^{2}$. We expect a second order phase transition when $f_{\mathrm{II}}$ changes sign. This happens for $(\Delta L / L)=(\Delta L / L)_{0}$ where

$$
\left(\frac{\Delta L}{L}\right)_{0}=\frac{K_{1}}{\bar{B}} \cdot k^{2}+\frac{\gamma}{\bar{B} d}+\frac{\varrho g}{B} d \cdot \frac{1}{k^{2}}
$$

as a function of $k$ has a minimum for the critical wavenumber $k_{\mathrm{c}}$ :

$k_{\mathrm{c}}=\left(\varrho g / K_{1} d\right)^{1 / 4} \quad$ or $\quad \lambda_{\mathrm{c}}=2 \pi\left(K_{1} d / \varrho g\right)^{1 / 4}$.

That is the wavelenght of the spontaneous rippling as soon as $(\Delta L / L)$ surpasses the critical value

$$
\left(\frac{\Delta L}{L}\right)_{\mathrm{c}}=\frac{1}{\bar{B} d}\left[\gamma+2\left(K_{1} \varrho g d\right)^{1 / 2}\right] \text {. }
$$

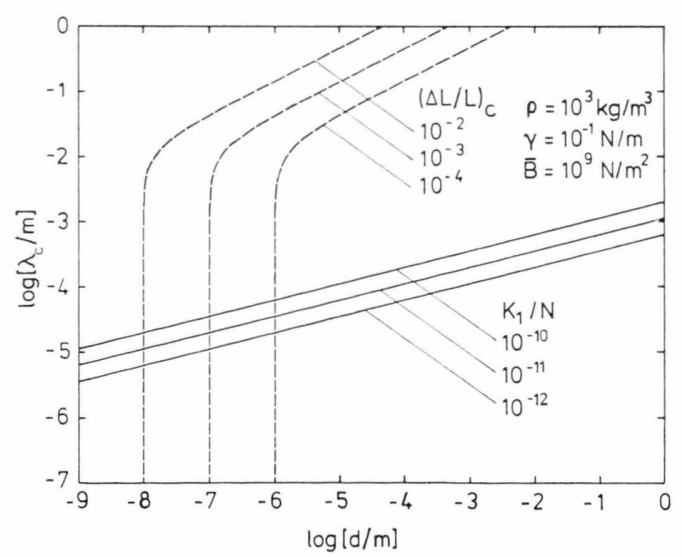

Fig. 2. Ripple wavelength $\lambda_{\mathrm{c}}$ vs skin thickness $d$ (log-log plot). Solid lines: Splay constant $K_{1}$ as parameter [see Equation (10)]. Dashed lines: Critical strain $(\Delta L / L)_{\mathrm{c}}$ as parameter [see Equation (12)].

In Fig. $2 \lambda_{\mathrm{c}}$ is plotted against $d$ from Eq. (10) on a double logarithmic scale (solid straight lines). For $\varrho$ the density of water has been chosen. $K_{1}$ is the free parameter. Below a thickness of about $10^{-4} \mathrm{~m} \lambda_{\mathrm{c}}$ remains larger than $d$. Down to a skin thickness of 
molecular dimensions $\lambda_{\mathrm{c}}$ should be observable in the microscope.

By using $K_{1}$ out of Eq. (10) to replace it in Eq. (11) one obtains

$$
\lambda_{c}{ }^{2}=\frac{2 \pi^{2}}{\varrho g}\left[\bar{B} d\left(\frac{\Delta L}{L}\right)_{\mathrm{c}}-\gamma\right] .
$$

The dashed curves in Fig. 2 show this relation between $\lambda_{\mathrm{c}}$ and $d$ when $(\Delta L / L)_{\mathrm{c}}$ acts as a free parameter. The other parameters have been reasonably fixed as $\bar{B}=10^{9} \mathrm{~N} / \mathrm{m}^{2}$ and $\gamma=10^{-1} \mathrm{~N} / \mathrm{m}$. In the region of the solid straight lines the dashed lines are parallel to the $\lambda_{\mathrm{c}}$ axis. In all practical cases $\gamma \gg\left(K_{1} \varrho g d\right)^{1 / 2}$ in Equation (11). Therefore a good approximation is

$$
(\Delta L / L)_{\mathrm{c}} \cong \gamma / \bar{B} d .
$$

For $d>10^{-6} \mathrm{~m}(\Delta L / L)_{\mathrm{c}}$ is sufficiently small so that plastic effects like the motion of dislocations into the skin will not take place before the rippling sets in. In order to avoid such difficulties in a thinner skin the surface tension $\gamma$ has to be reduced drastically.

The simultaneous action of a magnetic field in the $z$ or $x$ direction is shown to be inefficient in practice. Its contribution to $f_{\mathrm{II}}$ in Eq. (6) has the form $\pm \frac{1}{4}\left(\mu_{0} \chi_{\mathrm{a}} H^{2} d\right) k^{2}$ and formally it acts in the same way as the surface tension. Therefore in Eqs. (11), (12), and (13) $\gamma$ has to be replaced by $\left(\gamma \pm \mu_{0} \chi_{\mathrm{a}} H^{2} d\right)$. However for small skin thicknesses $d$ this magnetic term can be neglected for all practical fields.

As for the solid skin ${ }^{1}$, the critical wavelength of the smectic A skin is independent of the stationary amplitude $z_{0}$. This equilibrium amplitute is obtained from Eq. (4) by minimizing $f$ with respect to the amplitude $z_{0}: \partial f / \partial z_{0}^{2}=0$ gives $z_{0}^{2}=-f_{\mathrm{II}} / 2 f_{\mathrm{IV}}$ or

$$
z_{0}^{2}=8\left[\left(\frac{\Delta L}{L}\right)-\left(\frac{\Delta L}{L}\right)_{\mathrm{c}}\right] / 3 k_{\mathrm{c}}{ }^{2} \text {. }
$$

1 F. Fischer, Z. Naturforsch. 32 a, 33 [1977].

2 W. Helfrich, Appl. Phys. Lett. 17, 531 [1970]; J. P. Hurault, J. Chem. Phys. 59, 2068 [1973].

3 M. Delaye, G. Ribotta, and G. Durand, Phys. Lett. A 44, 139 [1973] ; N. Clark and R. B. Meyer, Appl. Phys. Lett. 22, 493 [1973].

4 F. Kahn, Appl. Phys. Lett. 22, 111 [1973].
With this amplitude and with $(\Delta L / L)_{\mathrm{c}}$ from Eqs. (9) and (11) put into Eq. (4), we find for the rippled skin an average free energy per unit area

$$
f_{1}=f_{0}-\frac{1}{3} \bar{B} d\left[\left(\frac{\Delta L}{L}\right)-\left(\frac{\Delta L}{L}\right)_{\mathrm{c}}\right]^{2}
$$

where

$$
f_{0}=\frac{1}{2} \bar{B} d(\Delta L / L)^{2}
$$

is the average free energy per unit-area for the plane skin. In Fig. $3 f_{0}$ and $f_{1}$ are plotted as a function of $(\Delta L / L)$. At $(\Delta L / L)$ c both curves meet without intersection. This is typical for a second order transition. The dashed part of $f_{1}$ is physically impossible because from (Eq. (14) the ripple amplitude would become imaginary. Experimental attempts to detect the periodic ripples and to prove the $1 / 4$ th power law between $\lambda_{\mathrm{e}}$ and $d$ have been initiated.

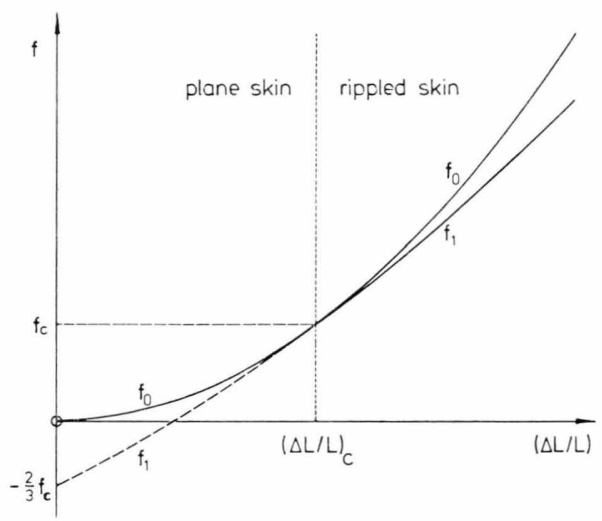

Fig. 3. Average free energy per unit-area $f$ as a function of compressive strain $(\Delta L / L) . f_{0}$ from Eq. (16), $f_{1}$ from Equation (15) .

I wish to thank Dr. C. B. Lucas for revising the manuscript.

5 P. G. de Gennes, J. Phys. 30, Colloq. C4 Suppt. to No. 11-12, 65 [1969]; P. G. de Gennes, The Physics of Liquid Crystals, Oxford 1974, p. 286.

6 J. Billard and W. Z. Urbach, C. r. Acad. Sci., Paris 274 B, 1287 [1972]; P. G. de Gennes, The Physics of Liquid Crystals, Oxford 1974, p. 292. 